\title{
Ensinando a Língua Estrangeira através de Gêneros Textuais: o resumo como uma atividade de retextualização
}

\author{
Teaching a Foreign Language through Textual Genres: \\ THE SUMMARY AS AN ACTIVITY OF RETEXTUALIZATION
}

Fabiano Silvestre RAMOS* Neuda Alves do LAGO**

Resumo: O objetivo do presente trabalho foi investigar quais são as concepções de resumo apresentadas por alunos universitários matriculados em um curso de língua estrangeira (LE) - Inglês, bem como verificar a maneira como se dá a produção do gênero resumo por tais alunos, através do processo de retextualização. Para tanto, foi aplicado um questionário com perguntas relacionadas à configuração do resumo, bem como foram analisadas as produções de resumos dos alunos em dois momentos distintos, antes e após a aplicação de uma sequência didática na qual o gênero foi ensinado de maneira sistemática. Utilizamos como embasamento teórico os estudos de Dell'Isola (2007) e Marcuschi (2005a) sobre retextualização, bem como uma abordagem de gêneros textuais proposta pelo interacionismo sócio-discursivo. Os resultados mostraram que a aplicação da sequência didática foi benéfica para os alunos em relação à produção de resumos, havendo grande melhora na escrita dos participantes em alguns aspectos. Porém, no que se refere à cópia

\footnotetext{
* Mestre em Letras (2011) pela Universidade Federal de Viçosa. Atualmente cursa o programa de Doutorado em Estudos Linguísticos pela Universidade Estadual Paulista "Júlio de Mesquita Filho". É Professor Assistente do Curso de Letras Inglês da Universidade Federal de Goiás - Regional Jataí. Contato: fabiano.silvestre.ramos@gmail.com.

** Doutora em Letras pela Universidade Federal de Goiás. Atualmente é Professora Adjunta do Curso de Letras Inglês da Universidade Federal de Goiás - Regional Jataí. Contato: neudalago@hotmail.com.
} 
de trechos do texto-base e à marcação de autoria, os alunos ainda apresentam problemas que merecem atenção.

Palavras-chave: Gêneros textuais. Resumo. Ensino de inglês. Retextualização.

Abstract: The aim of this paper was to investigate the conceptions of summary presented by some university students enrolled in an English language course, as well as to verify how those students produced this genre, through retextualization. In order to do so, we used a questionnaire with a set of questions related to the summary structure, and analyzed the participants' productions of summaries in two distinct moments: before and after the use of a didactic sequence which taught that text genre systematically. The theoretical foundations of this work are based on the studies of scholars such as Dell'Isola (2007) and Marcuschi (2005a), concerning retextualization, as well as on a socio-discursive interactionist view of genres. The results indicate that the use of the didactic sequence was beneficial for the students regarding their summary productions, as there was improvement in their writing in some aspects. Nevertheless, concerning the copies of extracts from the basic text and the marks of authorship, the participants presented some issues which deserve further attention.

Key words: Textual genres. Summary. English teaching. Retextualization.

\section{Introdução}

O ensino de línguas na contemporaneidade exige uma nova configuração do sistema educacional, assim como novas posturas dos atores sociais envolvidos em tal contexto. Como afirma Xavier (2010, p. 75-76), “o ensino atual assume, ou deveria assumir, características diferentes do ensino de épocas passadas, mesmo porque estamos vivendo em um outro momento histórico." Dessa forma, o ensino de estruturas gramaticais por si só, bem como o ensino de frases soltas, em metodologias vendidas como comunicativas, não satisfaz mais o aprendiz, consumidor de informações. Faz-se mister a busca de outros caminhos para o ensino, numa abordagem mais ampla, que contemple o estado do homem atual.

O ensino baseado em gêneros discursivos vem suprir essa necessidade. A comunicação humana acontece através do uso dos mais variados tipos de enunciados, que, relativamente estáveis, se configuram em gêneros. O 
conhecimento desses gêneros, dessa forma, empodera o indivíduo, nos termos faircloughianos, a ter uma atuação mais efetiva na comunidade da qual faz parte.

Pretendemos, com este trabalho, analisar a forma como alunos universitários de um curso de extensão em língua inglesa se apropriam do gênero resumo, parte constitutiva do discurso científico da comunidade discursiva desses participantes. O interesse por essa pesquisa partiu de nossa prática enquanto professores que lidam com línguas e literaturas estrangeiras. Discordamos de metodologias que pregam o ensino da fala como prioridade para que, somente depois que o indivíduo for proficiente na parte oral da língua, possa se trabalhar a escrita ${ }^{1}$. Faz-se, em tais metodologias, uma analogia com o aprendizado de língua materna, cujo processo de aquisição se dá primeiramente através da fala. Por se tratar de um processo distinto, acreditamos em um trabalho conjunto entre fala e escrita nas aulas de língua estrangeira.

Levamos em consideração, neste trabalho, as orientações dos Parâmetros Curriculares Nacionais (PCNs) (BRASIL, 1998) de que o ensino deve ser baseado na produção e compreensão de gêneros discursivos. Os PCNs trazem uma proposta de linguagem, sob uma perspectiva sócio-histórica, na qual se dá a produção dos gêneros discursivos. Deve-se, dessa forma, refletir sobre o uso da linguagem para que o aluno compreenda e a empregue nas mais diversas situações. Admitindo isso, a unidade básica do ensino deve ser o texto ativo, construído em situações de comunicação com propósitos específicos, apresentando estilo e temas adequados ao contexto.

Portanto, no presente trabalho, investigamos como se dá a produção do gênero resumo por alunos de um curso de língua inglesa através do processo de retextualização. Analisamos as produções em dois momentos distintos: antes da aplicação de uma sequência didática, na qual o gênero resumo escolar foi trabalhado, e após sua aplicação. Comparamos as duas produções para verificar de que maneira o ensino sistemático de tal gênero modificou a prática de produção dos participantes. O trabalho com o gênero

${ }^{1}$ Utilizamos, neste trabalho, as concepções de fala e escrita como modalidades de uso da língua, conforme pontuado por Marcuschi (2005a), sem, contudo, preconizar a prioridade daquela em detrimento desta, no contexto de ensino e aprendizagem de língua estrangeira. 
resumo justifica-se devido a ser este um dos principais gêneros acadêmicos na sociedade atual. Essa importância assumida por tal gênero tem levado vários estudos a se dedicarem a essa temática (cf. RAMOS; GOMES, 2009).

$\mathrm{Na}$ primeira parte deste trabalho, expomos as teorias que embasaram o estudo com o gênero resumo em sala de aula. Em seguida, discorremos sobre os procedimentos metodológicos desta pesquisa, para, então, apresentar uma análise dos dados obtidos e algumas considerações finais.

\section{Pressupostos Teóricos}

\subsection{Gêneros textuais}

Muitos estudos têm sido dedicados à investigação dos gêneros nos últimos anos, sob diferentes perspectivas e abordagens, constituindo o que chamamos de diferentes escolas, dentre elas a norte-americana, a australiana e a de Genebra. Porém, como afirma Ramires (2005, p. 2), "pode ser constatado como um dos pontos comuns entre eles o fato de reconhecerem explicitamente a primazia do social na compreensão dos gêneros e no papel do contexto". Trabalhamos neste artigo com a visão de gêneros textuais proposta pelo interacionismo sociodiscursivo (ISD). Segundo Ramires (2005), dentre as correntes teóricas acerca dos gêneros textuais, a que mais está voltada para a questão do ensino de língua materna é aquela adotada pela chamada Escola de Genebra, que tem como representantes mais significativos Bernard Schneuwly, Joaquim Dolz e Jean-Paul Bronckart. No Brasil, podemos listar alguns autores que trabalham dentro dessa abordagem, tais como Cristovão (2005), Machado (2005) e Marcuschi (2008), autores que embasarão este referencial teórico.

De acordo com Machado (2005, p. 238), o ISD não entende os gêneros textuais

... como sua unidade de análise privilegiada nem considera que sua análise seja seu objetivo maior. De fato, as unidades de estudo privilegiadas são as ações verbais e não-verbais, sendo os objetivos dessa análise dirigidos por concepções epistemológicas específicas.

Uma perspectiva comum aos autores que se agrupam na ISD é a preocupação com a intervenção no contexto educacional. Volta-se para a 
atividade de ensino e aprendizagem de língua materna e/ou estrangeira. Um estudo que ilustra essa perspectiva é o de Ramos e Gomes (2009), que pesquisam o ensino do gênero resumo no primeiro ciclo do ensino fundamental, a partir da aplicação de um curso de intervenção na produção desse gênero acadêmico.

Considerando essa perspectiva, adotamos a definição de Marcuschi (2008, p. 155), que entende gêneros textuais como sendo

... os textos que encontramos em nossa vida diária e que apresentam padrões sociocomunicativos característicos definidos por composições funcionais, objetivos enunciativos e estilos concretamente realizados na integração de forças históricas, sociais, institucionais e técnicas.

Segundo Bakhtin (2000, p. 279), toda atividade humana é mediada pelo uso da língua, que acontece pelo uso de enunciados "concretos e únicos, que emanam dos integrantes de uma ou outra esfera da atividade humana". A contribuição de Bakhtin para os estudos de gêneros é de grande importância. Como sugere Ramires (2005), não é possível se falar em gêneros textuais sem mencionar o trabalho de Bakhtin, Estética da criação verbal (2000).

Machado (2005, p. 250), baseando-se em Bronckart (2001), afirma que os gêneros textuais são pré-construtos, em outras palavras, "construtos existentes antes de nossas ações, necessários para sua realização". Entendendo toda atividade humana como sendo mediada pelo uso da linguagem, podemos compreender os gêneros como instrumentos de mediação de nossas ações. Para Machado (2005, p. 250), os gêneros encontram-se indexados a situações de ação da linguagem, sendo portadores de valores de uso, ou seja, "em uma determinada formação, determinado gênero é considerado como mais ou menos pertinente para determinada ação".

De acordo com Bronckart (1999, p. 103), “a apropriação dos gêneros é um mecanismo fundamental de socialização, de inserção prática nas atividades comunicativas humanas". Uma vez que toda comunicação humana acontece por mediação dos gêneros textuais, pensar em uma abordagem de ensino baseada neles é de fundamental importância. Assim como o ensino de gêneros acadêmicos, por exemplo, que os aprendizes irão se empoderar desses para uma atuação efetiva na comunidade discursiva da qual fazem parte.

Na concepção de Lee (2001), da qual também partilhamos, trata-se de uma tarefa irrealizável o fazer generalizações úteis sobre a língua inglesa ou sobre o inglês, de forma geral, dado o alto grau de abstração desses construtos. 
Por outro lado, é possível realizar um trabalho profícuo, e muito melhor fundamentado teoricamente, com "a linguagem usada em distintos gêneros de texto, ou as linguagens utilizadas em diferentes domínios, ou os diferentes tipos de registro disponíveis em uma língua, e assim por diante"2 (LEE, 2001, p. 38). Para o autor, o "gênero é o nível de categorização textual mais útil e prático de se trabalhar, teórica e pedagogicamente"3 (LEE, 2001, p. 37), o que também justifica o enfoque dado a ele neste trabalho.

\section{$1.2 O$ gênero resumo como uma atividade de retextualização}

$\mathrm{O}$ ato de resumir é uma prática muito comum em nossas vidas. Resumimos o fato acontecido para alguém conhecido, resumimos uma fofoca que escutamos, uma novela que assistimos. Da mesma forma, ele está presente também na academia, como um dos principais gêneros acadêmicos. Assim, como afirmam Ramos e Gomes (2009), o ensino desse gênero pode auxiliar o aprendiz no processo de inclusão ou ascensão social, sendo que a sociedade exige cada vez mais, dos seus cidadãos, um conhecimento de uma vasta quantidade de gêneros discursivos. No contexto acadêmico, onde o resumo é prática recorrente, é de fundamental importância que os aprendizes o dominem. Para que possa atuar de maneira eficaz nos diferentes contextos da sociedade, é necessário que o indivíduo domine os gêneros utilizados na comunicação naquele contexto específico. Entender o gênero dessa forma seria concordar com Miller (1984) e sua proposta de gênero como forma de ação social. Conhecer e ser capaz de reconhecer os gêneros utilizados em determinada comunidade discursiva faria com que o indivíduo pudesse nela se inserir, aumentando suas oportunidades de inclusão ou ascensão social.

O ensino da habilidade da escrita em língua estrangeira tem estado em foco como uma das habilidades mais importantes nesse campo de aprendizagem, cobrindo uma vasta gama de propostas didáticas. Embora alguns considerem a tentativa de ensinar a escrita como inócua, asseverando que "a empreitada toda está muito além das palavras - além de qualquer

2 ... the language of different genres of text, or the language(s) used in different domains, or the different types of register available in a language, and so forth. (LEE, 2001, p. 38, tradução nossa).

${ }^{3}$ [G]enre is the level of text categorisation which is theoretically and pedagogically most useful and most practical to work with. (LEE, 2001, p. 37, tradução nossa). 
concepção"4 (SMITH, 1982, p. 27), várias foram as técnicas criadas e defendidas como as mais eficazes neste propósito. Tais técnicas e procedimento se alistaram, dominantemente, até duas décadas atrás, no que Badger e White (2000, p. 153) chamaram de abordagens de processo e produto: "abordagens de processo e produto dominaram a maior parte do ensino de escrita que acontece na sala de aula de inglês como língua estrangeira". ${ }^{5}$ As abordagens de processo enfatizavam as fases da escrita, enquanto as abordagens de produto ressaltavam o resultado da organização de ideias, na facção final de um texto. Porém, como atentam os autores, desde a década de 90 surgiu um novo paradigma no ensino de escrita: a abordagem de gêneros, que derivava da abordagem de produto.

As abordagens de gênero são relativamente novas na área de Ensino de Língua Inglesa. Contudo, há fortes semelhanças com as abordagens de produto e, em certos aspectos, as abordagens de gênero podem ser consideradas como uma extensão das abordagens de produto. Como elas, as abordagens de gênero consideram a escrita como predominantemente lingüística, mas, distintamente das abordagens de produto, enfatizam que a escrita varia de acordo com o contexto social em que é produzida. ${ }^{6}$ (BADGERS; WHITE, 2000, p. 155)

Este trabalho se afilia dominantemente à abordagem de gêneros, tendo como foco o gênero resumo ${ }^{7}$.

${ }^{4}$ The whole enterprise is beyond words - beyond conception. (SMITH, 1982, p. 27, tradução nossa).

${ }^{5}$ Process and product approaches have dominated much of the teaching of writing that happens in the EFL classroom. (BADGER; WHITE, 2000, p. 153, tradução nossa).

${ }^{6}$ Genre approaches are relative newcomers to ELT. However, there are strong similarities with product approaches and, in some ways, genre approaches can be regarded as an extension of product approaches. Like product approaches, genre approaches regard writing as predominantly linguistic but, unlike product approaches, they emphasize that writing varies with the social context in which it is produced. (BADGERS; WHITE, 2000, p. 155, tradução nossa).

${ }^{7}$ Como Badger e White (2000), porém, concordamos em que as três abordagens de ensino de escrita mencionadas (de processo, de produto e de gêneros) são complementares, e têm muito a oferecer umas às outras. 
Segundo Costa (2008, p. 160), o resumo pode ser uma apresentação abreviada de um texto, do conteúdo todo de um livro, de uma peça teatral, do argumento de um filme, etc.. Podemos falar de resumo em termos de redução de informação (LEITE, 2006, p. 11), sendo, assim, resultado de um processo de compreensão de um texto-base. Conforme ressalta a autora, só conseguiremos resumir aquilo que entendermos de maneira efetiva. Como aponta Marcuschi (2005a, p. 59), a atividade de produção de resumos é essencial para se trabalhar a compreensão de textos, visto que, através da produção do resumo, o aluno desenvolverá a interpretação global do texto, sendo que, para resumir, o indivíduo deve, primeiramente, compreender o texto-base e acionar sua capacidade de percepção dos seus aspectos mais importantes.

Machado, Lousada e Abreu-Tardelli (2004) e Leite (2006) propõem etapas para a produção de resumos que consistem em duas estratégias: seleção e construção. A primeira diz respeito à triagem do conteúdo lido, em busca de informações relevantes. Nesse momento, ocorre a cópia do que será utilizado e o apagamento de informações desnecessárias. A segunda etapa consiste na reconstrução do conteúdo selecionado. Após a seleção das informações que serão consideradas na produção do resumo, estas serão reconstruídas por meio de paráfrase ou de um processo de generalização, na qual "uma seqüência que encerra informações particulares pode ser substituída por itens que a englobe [...]" ou, ainda, por meio da construção, pela qual “[...] uma seqüência ampla de informações pode ser substituída por outra” (LEITE, 2006, p. 17).

Nossa memória seleciona informações as quais julga serem mais relevantes e apaga outras, consideradas de menor importância. Porém, essas ações são movidas por motivações individuais (LEITE, 2006). A autora ressalta que isso causa alguns problemas no instante da produção do resumo. É de conhecimento geral, em se tratando de textos escritos, que eles apresentam basicamente uma finalidade comunicativa, ou seja, todo texto tem um propósito de informar algo a alguém. Sendo assim, é evidente que possuem um núcleo de informações "universalmente" consideradas mais importantes e outras nem tanto, não cabendo ao leitor escolher por si só o que acredita ser ou não relevante. Dessa forma, o processo de resumir não pode ser inteiramente subjetivo. Considerando tais fatos, é possível afirmar que um bom resultado, no que concerne ao resumo, dependerá das competências de leitura do indivíduo. 
Como pontuado por Machado, Lousada e Abreu-Tardelli (2004), o resumo deve obedecer à organização do texto-base, mantendo as relações entre as ideias estabelecidas pelo autor, e isso pode ser alcançado por meio do uso de conectivos ou organizadores textuais.

\subsection{Falando um pouco sobre retextualização}

Utilizamos, neste trabalho, a abordagem de retextualização de gêneros escritos proposta por Dell'Isola (2007), que define retextualização como sendo uma refacção ou reescrita de um texto em um novo texto, de um gênero em um outro gênero. Para a autora, "trata-se de um processo de transformação de uma modalidade textual em outra, envolvendo operações específicas de acordo com o funcionamento da linguagem" (DELL'ISOLA, 2007, p. 36).

Travaglia (1993) utilizou o termo retextualização para se referir à atividade de tradução, numa abordagem textual daquela atividade. Como o conceito tradicional de tradução evoca, de forma geral, uma noção primária de duas línguas envolvidas (conceito que tem sido ampliado consideravelmente, nas últimas décadas, para incluir mudanças entre sistemas semióticos, gêneros etc.), a autora, em sua tese de doutoramento, utilizou a retextualização para abordar a transformação feita no processo de transpor de uma língua para outra: numa perspectiva textual, traduzir seria retextualizar, reenunciar. Ela afirmou que os elementos responsáveis pela textualidade de um texto foram acionados pelo escritor do texto original, e esses elementos, sob uma ótica distinta, são trabalhados pelo tradutor, assim como elementos distintos, da outra língua, para a qual o texto estaria sendo traduzido. O processo apresentase, então, como mais complexo do que uma mera recodificação, visto que engloba as novas condições de produção, sob uma perspectiva discursiva.

Retomando aquele conceito, Marcuschi (2005a, p. 46) incorpora a ele uma dimensão mais ampliada, envolvendo, agora, ações intralinguais: "[o] uso do termo retextualização, tal como feito aqui, se recobre apenas parcialmente com aquele feito por Travaglia, na medida em que aqui também se trata de uma 'tradução', mas de uma modalidade para outra, permanecendo-se, no entanto, na mesma língua”.

Nesse trabalho, o enfoque de Marchuschi (2005a) são as relações entre as duas modalidades da língua: falada e escrita. Ele analisa textos orais retextualizados para textos escritos, em situações concretas. Sua posição favorece a desmistificação de uma supremacia alegada da modalidade escrita 
da língua, em detrimento da falada. Com esse propósito, Marcuschi (2005a, p. 47-48) argumenta que "a retextualização não é, no plano da cognição, uma atividade de transformar um suposto pensamento concreto em um suposto pensamento abstrato". O autor afirma que as atividades de retextualização são "rotinas usuais altamente automatizadas, mas não mecânicas, que se apresentam como ações aparentemente não-problemáticas” (MARCUSCHI, 2005a, p. 48).

Dell'Isola (2007) concorda com esse caráter ordinário da retextualização, ressaltado por Marchuschi (2005a), já que é uma atividade muito presente em nosso cotidiano, uma vez que estamos a todo momento transformando um gênero em outro, como quando fazemos uma sinopse oral de um filme que assistimos recentemente, por exemplo. Entretanto, de forma distinta da trabalhada por Marcuschi (2005a), que enfatizou situações concretas em que textos da modalidade oral foram retextualizados para a escrita, Dell'Isola (2007) foca em operações de retextualização na esfera dos gêneros escritos. Mais especificamente, o estudo da autora se debruça sobre momentos em que um texto escrito é utilizado como base para outra produção também escrita, se configurando na passagem de um gênero escrito para outro gênero, também escrito, no contexto do ensino de língua, em sala de aula. Segundo ela, a retextualização

... é um processo que envolve operações complexas que interferem tanto no código como no sentido e evidencia uma série de aspectos da relação entre oralidade-escrita, oralidade-oralidade, escrita-escrita, escrita-oralidade. Retextualização é a refacção ou a reescrita de um texto para outro, ou seja, trata-se de um processo de transformação de uma modalidade textual em outra, envolvendo operações específicas de acordo com o funcionamento da linguagem. (DELL'ISOLA, 2007, p. 36).

Segundo a autora, podemos dizer que o processo de retextualização é um dos mais comuns na área de comunicação. Como Marchuschi, Dell'Isola (2007) defende que, exatamente por se tratar de um dos atos mais utilizados nas relações humanas, a retextualização não pode ser considerada com artificialidade. Tomando como exemplos situações advindas de eventos corriqueiros da vida social e que ilustram as operações de retextualizações de textos, a autora reforça o fato de que os exemplos apresentados se configuram 
como prova de "que um mesmo conteúdo pode ser retextualizado de muitas maneiras" (DELL'ISOLA, 2007, p. 37). Em cada instância de retextualização de um texto em um novo texto, temos o mesmo dito de outra forma, distinta da anterior, criando, assim uma nova situação comunicativa, que traz em seu bojo novos propósitos específicos.

Marcuschi (2005a) apresenta quatro possibilidades de retextualização, como podemos verificar no quadro abaixo, proposto pelo autor:

\begin{tabular}{|lll|}
\hline \multicolumn{2}{l|}{ Quadro 1. Possibilidades de retextualização } \\
\hline 1. Fala & $\rightarrow$ & Escrita (entrevista oral $\rightarrow$ entrevista impressa) \\
2. Fala & $\rightarrow$ & Fala (conferência $\rightarrow$ tradução simultânea) \\
3. Escrita & $\rightarrow$ & Fala (texto escrito $\rightarrow$ exposição oral) \\
4. Escrita & $\rightarrow$ & Escrita (texto escrito $\rightarrow$ resumo escrito) \\
\hline
\end{tabular}

Fonte: Marcuschi, 2005a, p. 48.

Como afirmam Andrade, Machado e Silva (2006), a atividade de retextualização é um meio muito útil para a avaliação e revisão de textos por parte dos alunos. Para desenvolver tal atividade, o aprendiz deve ter em mente objetivos claros quanto ao gênero em que irá resultar no final, bem como quanto à finalidade comunicativa desse gênero. Conforme afirma Bakhtin (2000, p. 301), o "intuito discursivo do locutor, sem que este renuncie à sua individualidade e à sua subjetividade, adapta-se e ajusta-se ao gênero escolhido, compõe-se e desenvolve-se na forma do gênero determinado". O trabalho de retextualização envolve diretamente a compreensão do texto lido, para que, assim, se dê a sua transformação em outro tipo de texto. Dessa forma, podemos afirmar que esse seria um tipo ideal de atividade a ser trabalhada em aulas de línguas (tanto maternas quanto estrangeiras).

\section{Metodologia}

Procedemos, neste trabalho, a uma pesquisa quantitativo-qualitativa, de cunho descritivo. O estudo foi realizado em um curso de extensão em língua inglesa, localizado em uma universidade federal da região sudeste do país. Tal curso foi criado para atender às necessidades da comunidade universitária no que diz respeito ao ensino de língua estrangeira. O curso é oferecido por estudantes de Letras a um preço módico. 
Participaram desta pesquisa 14 alunos matriculados em uma turma de nível intermediário (que equivale a seis semestres de curso). A idade média dos participantes é de 20 a 27 anos. A turma é composta por alunos pertencentes às quatro áreas de conhecimento da universidade: Agrárias, Biológicas, Humanas e Exatas.

O gênero resumo foi trabalhado através de uma sequência didática que, segundo Dolz, Noverraz e Schneuwly (2004, p. 97), “tem, precisamente, a finalidade de ajudar o aluno a dominar melhor um gênero de texto, permitindo-lhe, assim, escrever ou falar de uma maneira adequada numa dada situação". Foi seguido o esquema de sequência didática por eles proposto, que apresenta a seguinte estrutura:

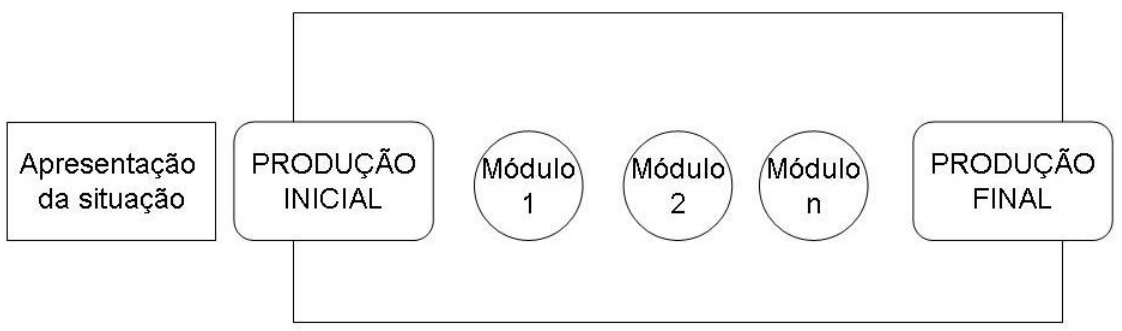

Fonte: Dolz, Noverraz e Schneuwly, 2004, p. 98.

Em um primeiro momento, foi pedido que os participantes respondessem a um questionário aberto que tinha como intuito verificar o que eles entendiam por fazer um resumo, bem como saber se esse gênero já havia sido trabalhado sistematicamente em sala de aula. Então, o gênero resumo foi apresentado para os alunos, ao mesmo tempo em que houve uma tentativa de conscientização dos alunos sobre a importância de tal gênero para a vida acadêmica deles. Nesse momento, os alunos, após uma discussão sobre o texto "Language after stroke" (a seguir), produziram um primeiro resumo. Dez alunos participaram desse primeiro momento da pesquisa. 


\section{Language after stroke}

Stroke sufferers often lose the ability to speak or remember events, but how difficult or easy it is to re-learn language can depend on which part of the brain was affected by the stroke and which language is being learnt.

Professor Anthony Kong from the University of Central Florida noticed this when working in Singapore. He found a big difference in stroke patients' symptoms depending on whether they spoke English or Chinese.

\section{Professor Kong}

In Chinese there is a higher portion of right hemisphere activation, in terms of processing the language, and this contrasts with English or other Latin based languages in which most of the activations are on the left side of the brain.'

He says brain imaging techniques show tonal languages, such as Chinese, make more use of the right side of the brain, whereas the processing for Latin based languages, such as English, tends to occur in the left side. This means for Chinese speakers, most of the medical research on re-learning language after a stroke may not be relevant to them, as it comes from investigations with English speakers.

Professor Kong is now about to start the first Chinese specific research project on the way brain damage affects speech. He hopes this will lead to better after stroke therapy for speakers of Chinese and other tonal languages.

Julian Siddle, BBC

Disponível em: <http://www.bbc.co.uk/worldservice/ learningenglish/language/wordsinthenews/009/10/091 020_witn_stroke_language.shtml >. Acesso em: 19 nov. 2009.

Após essa primeira produção, o gênero resumo foi trabalhado sistematicamente com os alunos através de dois módulos. No primeiro módulo, os alunos tiveram contato com as características do gênero resumo, seu 
funcionamento e as estratégias de sua feitura. Foram apresentados, aos aprendizes, diversos tipos de resumo, nos quais eles puderam identificar, por eles mesmos, quais as características recorrentes em todos os textos para que, assim, se pudesse chegar à configuração do gênero em questão. As atividades utilizadas nessa aula foram adaptadas de Machado, Lousada e Abreu-Tardelli (2004) (cf. Anexo 1).

No segundo módulo, os alunos produziram um resumo coletivamente, utilizando as estratégias propostas por Leite (2006) e Machado, Lousada e Abreu-Tardelli (2004) (cf. Anexo 2). Por fim, eles escreveram um resumo do mesmo texto utilizado para a primeira produção, dessa vez conhecendo a configuração do gênero. Quatorze alunos participaram dessa segunda etapa da pesquisa.

O que difere na sequência por nós aplicada do modelo proposto por Dolz, Noverraz e Schneuwly (2004) foi o fato de que os alunos não tiveram acesso ao texto inicial corrigido, para que a segunda produção não se tornasse apenas uma reescrita da primeira.

\section{Análise dos Dados}

Discorremos, nesta seção, sobre os resultados da análise do questionário aplicado e das produções dos participantes antes e após a aplicação da sequência didática.

\subsection{A concepção de alunos universitários sobre o gênero resumo}

Apresentamos, inicialmente, os resultados do questionário aplicado com a finalidade de se conhecer as concepções dos participantes deste estudo sobre o gênero resumo. Foi perguntado aos alunos o que eles entendiam por resumo, sobre suas características, e em que momento de suas vidas acadêmicas tal gênero foi ensinado.

As ideias de síntese e concisão apareceram com certa recorrência nas respostas dos alunos, como é possível perceber nos excertos a seguir, pela presença de termos como "abordagem sucinta", "síntese", "concentrar determinado texto em poucas palavras" e "ideia geral":

(1) Uma abordagem sucinta sobre um tema, com informações o suficiente para que se tenha consciência da amplitude do que é 
abordado, mas sendo necessário o estudo do tema para maiores informações e detalhes. (Exemplo 1 - questionário - Aluno A)

(2) Síntese de um texto, sem se fazer relação a outros textos. (Exemplo 2 - questionário - Aluno B)

(3) Concentrar determinado texto em poucas palavras, conservando sua essência e sem mudar a direção do original. (Exemplo 3 questionário - Aluno C)

(4) Texto que consiga expressar a ideia geral de um texto ou qualquer outro tipo de texto. (Exemplo 4 - questionário - Aluno D)

Chamamos a atenção, aqui, para alguns pontos que merecem destaque. No excerto (1), o aluno afirma que o resumo deve ter informações suficientes para a compreensão do que o texto trata, porém, não desobrigando a leitura do texto-base para que se tenha uma visão mais aprofundada sobre o assunto. Em (2), o participante afirma que o resumo não apresenta relações com outros textos que não o texto-base. No trecho (3), o aluno chama a atenção para o fato de que o resumo deve seguir a direção do texto original, obedecendo a sua estrutura, corroborando o que é apresentado por Machado, Lousada e Abreu-Tardelli (2004, p. 43). Em (4), o participante expõe uma informação muito relevante e verdadeira: a de que qualquer gênero discursivo pode ser resumido, seja ele oral ou escrito. É importante ter isso em mente porque, por não haver um trabalho sistematizado sobre o gênero resumo nas escolas regulares, os alunos apresentam muitas dificuldades em resumir textos, tabelas, etc..

Quanto ao aprendizado desse gênero, alguns alunos afirmaram que o resumo nunca fora ensinado sistematicamente durante sua vida acadêmica. Dois alunos mencionaram que o resumo foi ensinado no primeiro semestre do curso de graduação, devido ao fato de terem uma grande carga de leituras a serem feitas e, nesse caso, o processo de sumarização dos textos seria de grande valia. Um aluno afirmou que o resumo foi trabalhado no ensino médio da seguinte forma:

(5) Foi ensinado no ensino médio, nas aulas de redação. Utilizou-se do livro de português onde havia resumos progressivos de textos e 
dicas de que tipos de ideias seriam melhor utilizadas ao resumir e nos era pedido fazer o semelhante aos exemplos de texto, ou limitando o número de linhas que o resumo poderia ter. (Exemplo 5 - questionário - Aluno C)

O trabalho com o resumo, no caso em questão, foi realizado a partir dos modelos propostos pelo livro didático, modelos esses que os alunos deveriam seguir como base na produção de seus próprios resumos. $\mathrm{O}$ tamanho da produção era limitado a certo número de linhas.

Através do questionário, foi possível perceber que os alunos possuíam um conhecimento relativo do que viria a ser o gênero resumo. Porém, na prática, como se daria a construção do resumo por tais alunos? $\mathrm{Na}$ seção seguinte, analisamos as produções dos participantes antes e após a aplicação da sequência didática. Nosso enfoque, neste trabalho, se dará mais na comparação, visto que objetivamos evidenciar os resultados do desenvolvimento da sequência didática. Assim, quanto aos dois momentos de escrita, apresentaremos um quadro geral das características que marcaram as produções do grupo, como um todo, não discorrendo sobre exemplos individualizados. Comparamos, em seguida, os resultados das produções realizadas nos dois momentos, com explicitação de exemplos elucidativos do processo de retextualização.

\subsection{Resultados da primeira produção}

Os participantes foram solicitados a resumir o texto "Language after stroke” (cf. seção de Metodologia). Durante a análise das primeiras produções, foi possível perceber que parte dos alunos apresentaram, em seus textos, características do gênero resumo, como exposto no quadro a seguir.

Pelos dados expostos, pode-se verificar que, embora os participantes tenham seguido vários aspectos componentes do gênero resumo, muitos outros quesitos, igualmente relevantes, não foram observados. 


\begin{tabular}{|c|c|c|c|}
\hline Característica & Sim & Não & Comentários \\
\hline Título & $50 \%$ & $50 \%$ & $\begin{array}{l}\text { Metade dos participantes atentou para } \\
\text { a necessidade de se colocar um título para o } \\
\text { resumo, usando o título do texto-base, } \\
\text { "Language after stroke". Nos textos que } \\
\text { não apresentaram título, não foi possível } \\
\text { identificar de qual texto os participantes } \\
\text { estavam tratando. }\end{array}$ \\
\hline Autoria & $20 \%$ & $80 \%$ & $\begin{array}{l}\text { Apenas dois alunos apresentaram, de certa } \\
\text { forma, a autoria do texto, ao iniciar o } \\
\text { resumo com a estrutura "The text talks } \\
\text { about...", ou "It explains that...". Os outros } \\
\text { textos apenas apresentaram as informações, } \\
\text { sem deixar claro que estas eram fornecidas } \\
\text { pelo autor do texto-base, tendo, portanto, } \\
\text { outra autoria que não a sua. }\end{array}$ \\
\hline $\begin{array}{l}\text { Estrutura do } \\
\text { texto-base }\end{array}$ & $100 \%$ & -- & $\begin{array}{l}\text { Todas as produções apresentaram a mesma } \\
\text { estrutura do texto-base. Possuíam uma } \\
\text { introdução, desenvolvimento das ideias e } \\
\text { uma pequena conclusão. Manteve-se } \\
\text { também o caráter informativo e de cunho de } \\
\text { divulgação científica do texto-base. }\end{array}$ \\
\hline Cópia & $40 \%$ & $60 \%$ & $\begin{array}{l}\text { Seis dos participantes fizeram cópia } \\
\text { ipsis literis de trechos do texto-base. Porém, } \\
\text { é importante mencionar que os casos de } \\
\text { cópia não se estendiam pelo texto todo, } \\
\text { apenas algumas partes do texto } \\
\text { apresentavam cópias do } \\
\text { texto-base. Desse número, somente uma } \\
\text { participante escreveu seu texto } \\
\text { completamente por meio de cópia. }\end{array}$ \\
\hline Concisão & $40 \%$ & $60 \%$ & $\begin{array}{l}\text { Seis participantes apresentaram, em seus } \\
\text { resumos, detalhes contendo informações } \\
\text { desnecessárias para a compreensão geral do } \\
\text { texto-base. }\end{array}$ \\
\hline $\begin{array}{l}\text { Apresenta } \\
\text { apenas } \\
\text { informações } \\
\text { presentes no } \\
\text { texto-base }\end{array}$ & $100 \%$ & -- & $\begin{array}{l}\text { Nenhuma produção apresentou } \\
\text { informações não presentes no } \\
\text { texto-base. }\end{array}$ \\
\hline $\begin{array}{l}\text { Apresenta } \\
\text { informações } \\
\text { suficientes para } \\
\text { a compreensão } \\
\text { do texto-base }\end{array}$ & $100 \%$ & -- & $\begin{array}{l}\text { Todas as produções apresentaram } \\
\text { informações suficientes para a } \\
\text { compreensão do texto-base (informações } \\
\text { tais como: o quê? onde? como? e por quê?) }\end{array}$ \\
\hline
\end{tabular}




\subsection{Resultados da segunda produção}

Apresentamos, a seguir, uma análise das produções de texto dos alunos após a aplicação da sequência didática, na qual foi trabalhado o gênero resumo.

Percebe-se, nesta segunda produção, um progresso no que concerne à observação dos requisitos do gênero resumo.

\begin{tabular}{|c|c|c|c|}
\hline Característica & Sim & Não & Comentários \\
\hline Título & $93 \%$ & $7 \%$ & $\begin{array}{l}\text { Apenas uma produção não apresentou } \\
\text { título. Das } 13 \text { que trouxeram título, } 11 \\
\text { repetiram o título do texto-base e } 2 \text { dos } \\
\text { participantes criaram outros títulos para o } \\
\text { seu resumo, baseando-se no tema principal } \\
\text { do texto de origem: "Chinese brain" e "Stroke } \\
\text { effects on language". }\end{array}$ \\
\hline Autoria & $50 \%$ & $50 \%$ & $\begin{array}{l}\text { Metade dos participantes marcou a autoria } \\
\text { do texto, utilizando a estrutura "The text } \\
\text { talks about...". A outra metade, mesmo } \\
\text { tendo sido alertada para essa necessidade de } \\
\text { deixar explícita a autoria do texto, não o fez. }\end{array}$ \\
\hline $\begin{array}{l}\text { Estrutura do } \\
\text { texto-base }\end{array}$ & $100 \%$ & -- & $\begin{array}{l}\text { Todos os participantes obedeceram a } \\
\text { estrutura do texto-base, que é um texto } \\
\text { descritivo de divulgação científica. }\end{array}$ \\
\hline Cópia & $35 \%$ & $65 \%$ & $\begin{array}{l}\text { Cinco participantes utilizaram a estratégia de } \\
\text { cópia na produção de seus resumos. Porém, } \\
\text { apenas alguns trechos foram copiados, } \\
\text { sendo que, na maioria do texto, foi utilizada } \\
\text { a paráfrase. }\end{array}$ \\
\hline Concisão & $29 \%$ & $71 \%$ & $\begin{array}{l}\text { Houve um aumento no número de } \\
\text { produções que utilizaram dados } \\
\text { desnecessários para a compreensão do } \\
\text { texto-base. O uso de exemplificações foi } \\
\text { encontrado em } 10 \text { das } 14 \text { produções } \\
\text { analisadas. }\end{array}$ \\
\hline $\begin{array}{l}\text { Apresenta } \\
\text { apenas } \\
\text { informações } \\
\text { presentes no } \\
\text { texto-base }\end{array}$ & $100 \%$ & -- & $\begin{array}{l}\text { Não houve acréscimo de informações não } \\
\text { presentes no texto-base. }\end{array}$ \\
\hline $\begin{array}{l}\text { Apresenta } \\
\text { informações } \\
\text { suficientes para } \\
\text { a compreensão } \\
\text { do texto-base }\end{array}$ & $100 \%$ & -- & $\begin{array}{l}\text { Todas as produções apresentaram } \\
\text { informações relevantes para a compreensão } \\
\text { geral do texto-base. }\end{array}$ \\
\hline
\end{tabular}




\subsection{Comparação entre as duas produções}

Foi possível perceber, nas segundas produções dos alunos, uma progressão em alguns aspectos de sua escrita, mas um retrocesso em outros. Houve um aumento significativo no número de participantes que apresentaram o uso de cópia idêntica e não explicitada de trechos do texto-base em suas produções. Porém, as partes em que o plágio foi conferido foram poucas. Predominou o uso de paráfrase das partes do texto original, como podemos perceber no exemplo abaixo:

Texto original: Professor Anthony Kong from the University of Central Florida noticed this when working in Singapore. He found a big difference in stroke patients' symptoms depending on whether they spoke English or Chinese.

Paráfrase: According to a research, the level of difficulty in re-learn depends on the language the patient speaks. (Exemplo 6 - segunda produção - aluno E)

No trecho anterior, pode-se perceber que a participante fez uma boa paráfrase do parágrafo original do texto-base. Houve, também, uma redução das informações desnecessárias à compreensão global do texto através do processo de generalização das informações presentes no original.

Em termos de autoria do texto, houve um aumento na explicitação da autoria do texto na produção do resumo. Consideramos de extrema importância que o indivíduo deixe claro, em seu texto, que as informações que ele está apresentando foram propostas por outra pessoa. Há algumas marcas linguísticas que podem ser utilizadas para delimitar essa autoria. Os participantes marcaram a autoria em seus resumos por meio da expressão "The text talks about...", como podemos perceber nos trechos abaixo:

The text talks about the difficulties of re-learning a language after a stroke, which can make people lose the ability to speak and forget things. (Exemplo 7 - segunda produção - aluno F)

The text talks about stroke sufferers and their ability to re-learn language -this depends if they spoke English or Chinese. (Exemplo 8 - segunda produção - aluno B) 
Faz-se mister ressaltar que, mesmo após o conhecimento da estrutura de um resumo, metade dos participantes não se preocupou em explicitar essa autoria. Os trechos abaixo referem-se ao parágrafo introdutório de algumas produções:

A researcher, Professor Anthony Kong, found on his researches that the tonal languages and Latin/English languages affects different brain sides. He hopes that the work with languages can be a therapy that can help stroke sufferers. (Exemplo 9 - segunda produção - aluno G)

Professor A. Kong found and noticed a big difference in the language re-learn of stroke sufferers: the difficulty on this process depends on which part of the brain was affected, related with which language is being learnt. (Exemplo 10 - segunda produção - aluno C)

Pode-se perceber, nos trechos anteriores, que os participantes introduziram as informações de forma direta, sem a menção da fonte, dando a impressão de que o texto é deles. É importante ressaltar, aqui, que, mesmo nos resumos em que houve explicitação da autoria, em nenhum momento os alunos mencionaram o nome do autor do texto, Julian Siddle, ou mesmo a palavra "author". A autoria de outrem, quando apareceu, foi marcada somente pela expressão "The text talks about".

Uma mudança importante em relação aos resumos produzidos nas duas etapas foi a diminuição no quesito concisão. Os participantes passaram a utilizar de exemplificações em seus resumos, algo que não pertence às características do gênero, e gera o efeito de expansão. Nos trechos a seguir, é possível perceber isso:

The symptoms are depending on the language the person speaks. For example, in Chinese there is a higher portion of the right hemisphere activation, while in the Latin languages most of the activation is in the left side of the brain, in terms of processing the language, and tonal languages. (Exemplo 11 - segunda produção - aluno $\mathrm{H}$ )

For example, a person that has less activity on the left brain side can work more with English or Latin languages. (Exemplo 12 - segunda produção - aluno I) 
Um aspecto que convém mencionar é que os participantes seguiram a mesma estrutura do texto-base, apresentando a clássica organização de introdução, desenvolvimento e conclusão. Não há acréscimo de informações não presentes no texto. Os participantes foram fiéis ao conteúdo do textobase. Isso mostra uma consciência a respeito dos tipos textuais, e de que o resumo de um artigo de divulgação científica, como é o caso do texto utilizado nesse experimento, deve manter o mesmo caráter do texto-base.

\section{Considerações (Quase) Finais}

Neste trabalho, fizemos uma investigação sobre as concepções de resumo apresentadas por alunos universitários matriculados em um curso livre de língua inglesa, bem como verificamos a maneira como se dá a produção do gênero resumo por tais alunos, através do processo de retextualização. Para tanto, aplicamos um questionário inicial, com perguntas relacionadas à configuração do resumo, e analisamos as produções de resumos dos alunos em dois momentos distintos: antes e após a aplicação de uma sequência didática, na qual esse gênero foi ensinado de maneira sistemática.

Por meio da análise dos questionários e das produções dos participantes desta pesquisa, pode-se afirmar que a maioria deles já dominava, de forma parcial, a configuração do gênero resumo. A concepção de que o resumo seria uma redução de informações de um texto-base era compartilhada por todos aqueles que participaram deste estudo. Porém, certas características, tais como a marcação da autoria do texto-base e a paráfrase de partes do texto, em vez da cópia, precisam ser reforçadas entre os sujeitos, uma vez que houve grande recorrência de problemas relacionados a elas nas produções dos participantes. Mesmo após um trabalho sistemático com o gênero, os alunos ainda não atentaram para tais características.

A maneira como o indivíduo compreende e interpreta um texto está diretamente relacionada ao resultado final do resumo produzido. Dessa forma, o trabalho com o gênero resumo se mostra muito eficaz para o professor ter acesso à maneira como o aprendiz está consumindo os textos propostos a ele.

Uma abordagem de ensino de línguas baseada em gêneros discursivos se mostra produtiva, uma vez que estamos preparando o indivíduo para a comunicação, tanto oral quanto escrita, nas mais variadas situações. Ao fazê-lo, estamos dando ao aluno conhecimento que irá auxiliá-lo em sua ascensão social, cumprindo, assim, com a verdadeira função do ensino. 


\section{Anexos}

\section{Anexo 1}

Activities adapted from: MACHADO, A. R.; LOUSADA, E.; ABREU-TARDELLI, L. S. Resumo. São Paulo: Parábola, 2004.

Exercise 1: Observe the texts below, paying attention to details such as layout, content and purpose and try to identify them, following the list given:

\section{Text 1:}

ABSTRACT: The purpose of this research was to evaluate the efficacy of a learning strategy intervention for the improvement of quality in narrative production. It was based on a quasi-experimental design with three stages: pretest, intervention sessions, and post-test. The quality of text production was examined by comparing two narratives (pre and post-test) in relation to text structure (presence of basic elements), level of idea articulation (hierarchy categories), spelling mistakes and number of written lines. The sample consisted of 35 6th grade students from a public school in Catalão-GO who were randomly distributed to experimental $(N=18)$ and control group $(N=17)$. The results showed that experimental group students produced better narratives in post-test characterized by adequate structure, good articulation of ideas and more written lines.

Keywords: Learning strategies; Text production; Teacher formation; Narratives.

COSTA, E. R.; BRUCHOVITCH, E. Learning style and narrative production. Psicologia: reflexão e crítica, v. 22, n. 2, p. 173-180, 2009. 


\section{Precious (Based on the novel PUSH, by Saphire)}

NOW A MAIOR MOTION PICTURE

p r e c i o u s

Based on the novel PUSH

by $\mathrm{s}$ a $\mathrm{p} \mathrm{ph}$ i $\mathrm{re}$

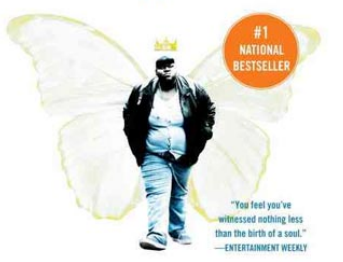

2009 Film Festival Award Winner

ce Grand Jury Prize • Toronto People's Choice Awart

Ineludes the novel Push and a reader's guide

Precious Jones, an illiterate sixteen-year-old, has up until now been invisible to the father who rapes her and the mother who batters her and to the authorities who dismiss her as just one more of Harlem's casualties. But when Precious, pregnant with a second child by her father, meets a determined and radical teacher, we follow her on a journey of education and enlightenment as she learns not only how to write about her life, but how to make it truly her own for the first time.

Text 3:

\section{Environmental protection targets missed}

Summary

3 May 2010

A major study has confirmed that the world's governments will not meet their internationally-agreed target of reducing the global loss of species and nature by 2010 .

\section{Report}

This study confirms what people in the conservation movement have known for several years; the 2010 target of significantly curbing the rate of biodiversity loss is not going to be met.

The researchers surveyed more than 30 trends covering wildlife on land and at sea. Virtually all of them show increasing degradation, with the evidence especially strong since the 1970s.

The reasons are straightforward; an ever-increasing number of people on the planet and rising affluence, leading to increased demand for food and timber and water and energy. As the human footprint expands, nature is squeezed to the margins. 
Governments set the 2010 target eight years ago; the researchers describe actions taken since then in order to meet it as "woefully inadequate". Richard Black, BBC Environment Correspondent

\section{Text 4:}

PENGUINS OF MADAGASCAR - Episode: 1x11 - "Crown fools" While trying to prove he can have fun on "Fun Day", Skipper causes Julien's crown to fall into the sewer, making Julien scream for hours. After a visit from an angry Maurice, the penguins set out to retrieve the crown. Meanwhile, Marlene tries to keep Julien calm, while he mourns for his cherished, missing crown. When the penguins find the crown, Skipper has to fight a giant rat for its return. When the crown is returned to Julien, he finds a smudge on it and calls for his spare crown. Furious, Marlene makes Rico regurgitate a crowbar so that she can beat up Julien.

\section{Text 5:}

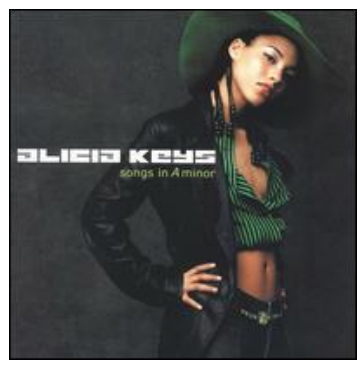

\section{SONGS IN AMINOR - Alicia Keys (2001)}

Alicia Keys' debut album, Songs in A Minor, made a significant impact upon its release in the summer of 2001, catapulting the young singer/songwriter to the front of the neo-soul pack. Critics and audiences were captivated by a 19 -year-old singer whose taste and influences ran back further than her years, encompassing everything from Prince to smooth '70s soul, even a little Billie Holiday. In retrospect, it was the idea of Alicia Keys that was as attractive as the record, since soul fans were hungering for a singer/songwriter who seemed part of the tradition without being as spacy as Macy Gray or as hippie mystic as Erykah Badu while being more reliable than Lauryn Hill. Keys was all that, and she had style to spare - elegant, sexy style accentuated by how she never oversang, giving the music a richer feel. It was rich enough to compensate for some thinness in the writing though it was a big hit, "Fallin' “ doesn't have much body to it - which is a 
testament to Keys' skills as a musician. And, the fact is, even though there are some slips in the writing, there aren't many, and the whole thing remains a startling assured, successful debut that deserved its immediate acclaim and is already aging nicely. $\sim$ Stephen Thomas Erlewine, All Music Guide

Source: Billboard <mmw.billboard.com>

( ) Summary of a TV series episode.

( ) Summary or abstract for a scientific paper.

( ) Album review.

( ) Summary of a book.

( ) Introduction (lead) of a news.

Exercise 2: Complete the table with the information from the texts below.

\begin{tabular}{|l|l|l|l|l|l|}
\hline & Text 1 & Text 2 & Text 3 & Text 4 & Text 5 \\
\hline Author & & & & & \\
\hline $\begin{array}{l}\text { Author } \\
\text { function }\end{array}$ & & & & & \\
\hline Addressee & & & & & \\
\hline $\begin{array}{l}\text { Media in } \\
\text { which you } \\
\text { can find } \\
\text { the text }\end{array}$ & & & & & \\
\hline $\begin{array}{l}\text { Aim of the } \\
\text { text }\end{array}$ & & & & & \\
\hline
\end{tabular}

Exercise 3: Complete the board with some of the characteristics of an academic summary.

\begin{tabular}{|l|l|}
\hline $\begin{array}{l}\text { Author of the } \\
\text { summary }\end{array}$ & \\
\hline Addressee & \\
\hline $\begin{array}{l}\text { Media in } \\
\text { which the text } \\
\text { will be shown. }\end{array}$ & \\
\hline Author's aim & \\
\hline
\end{tabular}


Exercise 4: Summarize the statements below, when possible. As you do it, point in the table the procedure you used for each statement.

a) Maria was a very good person. She loved helping people.

b) We will discuss about the structure of argumentative texts, e.g., those texts in which the author defends a certain point of view using for it some arguments, trying to convince the reader about his own point of view.

c) Don't run so fast because when you run this fast it is not possible to see the landscape and, besides this, the number of fatal accidents increase with the speed.

d) The prime suspect in the murder was her husband: he was jealous and had no alibi, since he states he has been watching the house to see if the woman was with her lover.

e) In the morning, he washed dishes, swept the house, dusted off and started laundry. In the afternoon, he went to the bank to pay bills, and in the evening, he prepared a lesson, corrected students' assignments and made a test.

f) The Enlightenment attacked injustice, religious intolerance and privileges typical of the old regime.

g) The death penalty has too many arguments in its favor, but nothing justifies taking the life of our fellow man.

h) In the summary of a narration, one can suppress the descriptions of place, time, persons or objects, if they are not necessary conditions for carrying out the action. For example, describing a man as jealous can be relevant and therefore, this description cannot be deleted since it is this quality that will determine that the man murdered his wife. But his description as tall and thin may be suppressed in this case.

( ) Deletion of content easily inferred from our knowledge of the world.

( ) Deletion of sequences of expressions that indicates synonyms or explanation.

( ) Deletion of examples.

( ) Deletion of the justifications for a claim.

( ) Deletion of the arguments against the position of the author.

( ) Reframing of information, using more generic terms (eg. man, cat, dog $\rightarrow$ mammals).

( ) Conservation of all information, given that they are not able to be summarized. 
Exercise: Summarize the text below to the minimum, considering that your addressee is your teacher and that he is going to evaluate your global comprehension of the text. Use the summary procedures studied previously.

\section{November 2009}

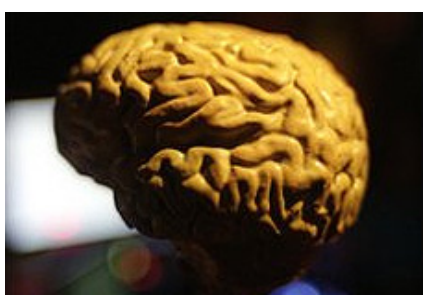

\section{Language after stroke}

A new initiative aimed at helping people re-learn language after suffering a stroke has shown the success of the learning may depend largely on which language the stroke sufferer is trying to learn.

Reporter: Julian Siddle

Stroke sufferers often lose the ability to speak or remember events, but how difficult or easy it is to re-learn language can depend on which part of the brain was affected by the stroke and which language is being learnt.

Professor Anthony Kong from the University of Central Florida noticed this when working in Singapore. He found a big difference in stroke patients' symptoms depending on whether they spoke English or Chinese. Professor Kong

'In Chinese there is a higher portion of right hemisphere activation, in terms of processing the language, and this contrasts with English or other Latin based languages in which most of the activations are on the left side of the brain.'

He says brain imaging techniques show tonal languages, such as Chinese, make more use of the right side of the brain, whereas the processing for Latin based languages, such as English, tends to occur in the left side. This means for Chinese speakers, most of the medical research on relearning language after a stroke may not be relevant to them, as it comes from investigations with English speakers.

Professor Kong is now about to start the first Chinese specific research project on the way brain damage affects speech. He hopes this will lead 
to better after stroke therapy for speakers of Chinese and other tonal languages.

Julian Siddle, BBC

Source: http://www.bbc.co.uk/worldservice/learningenglish/language/

wordsinthenews/2009/10/091020_witn_stroke_language.shtml

\section{Referências}

ANDRADE, E. P. de O.; MACHADO, G. dos S. S.; SILVA, S. R. Retextualização de uma história em quadrinhos por alunos de meios letrados. Linguagem \& Ensino, v. 9, n. 2, p. 177-199, jul./dez. 2006.

BADGER, R.; WHITE, G. A process genre approach to teaching writing. ELT Journal, v. 54, n. 2, p. 153-160, 2000.

BAKHTIN, M. Os gêneros do discurso. In: BAKHTIN, M. Estética da criação verbal. São Paulo: Martins Fontes, 2000. p. 277-326.

BRASIL. Ministério da Educação. Secretaria da Educação Média e Tecnológica. Parâmetros curriculares nacionais: ensino médio - linguagens, códigos e suas tecnologias. Brasília: MEC; SEMTEC, 1998.

BRONCKART, J.-P. Atividade de linguagem, textos e discursos: por um interacionismo sócio-discursivo. Tradução A. R. Machado. São Paulo: EDUC, 1999.

BRONCKART, J.-P. La psychologie ne peut être que sociale et la didactique est l'une de ses disciplines majeures. In: BERNIÉ, J.-P. (Org). Apprentissage, développement et signigications: hommage à Michel Brossard. Bordeaux: Presses Universitaires de Bordeaux, 2001. p. 19-41.

COSTA, S. R. Dicionário de gêneros textuais. Belo Horizonte: Autêntica, 2008.

CRISTOVÃO, V. L. L. Gêneros textuais, material didático e formação de professores. Signum: Estudos da Linguagem, Londrina, v. 8, n. 1, p. 173-191, jun. 2005.

DELL'ISOLA, R. L. P. Retextualização de gêneros escritos. Rio de Janeiro: Lucerna, 2007. 
DOLZ, J.; NOVERRAZ, M.; SCHNEUWLY, B. Seqüências didáticas para o oral e a escrita: apresentação de um procedimento. In: SCHNEUWLY, B.; DOLZ, J. (Orgs). Gêneros orais e escritos na escola. Campinas: Mercado de Letras, 2004. p. 95-127.

LEE, D. Y. Genres, registers, text types, domains, and styles: clarifying the concepts and navigating a path through the BNC jungle. Language Learning \& Technology, v. 5, n. 3. p. 37-72, 2001.

LEITE, M. Q. Resumo. São Paulo: Paulistana, 2006.

MACHADO, A. R. A perspectiva interacionista sociodiscursiva de Bronckart. In: MEURER, J. L.; BONINI, A.; MOTTA-ROTH, D. (Orgs.). Gêneros: teorias, métodos e debates. São Paulo: Parábola, 2005. p. 237-259.

MACHADO, A. R.; LOUSADA, E. G.; ABREU-TARDELLI, L. S. Resumo. São Paulo: Parábola, 2004.

MARCUSCHI, L. A. Da fala para a escrita: atividades de retextualização. 6. ed. São Paulo: Cortez, 2005a.

MARCUSCHI, L. A. Gêneros textuais: configuração, dinamicidade e circulação. In: KARWOSKI, A. M.; GAYDECZKA, B.; BRITO, K. S. Gêneros textuais: reflexões e ensino. Palmas; União da Vitória: Kaygangue, 2005b.

MARCUSCHI, L. A. Produção textual, análise de gêneros e compreensão. São Paulo: Parábola, 2008.

MILLER, C. R. Genre as social action. Quarterly Journal of Speech, n. 70, p. 151-67, 1984.

RAMIRES, V. Panorama dos estudos sobre gêneros textuais. Rev. Investigações, v. 18, n. 2, p. 1-28, jul. 2005. Disponível em: <http:// revistainvestigacoes.com.br/Volumes/Vol.18.N.2_2005_ARTIGOSWEB/ VicentinaRamires_PANORAMA-DOS-ESTUDOS_Vol18N2_Art02.pdf>. Acesso em: 26 nov. 2009.

RAMOS, F. S.; GOMES, M. C. A. Construindo experiências com a utilização do gênero resumo. Vertentes, São João Del-Rei, n. 34, p.133-143, jul./dez. 2009. 
SMITH, F. Writing and the writer. Londres: Heinemann, 1982.

TRAVAGLIA, N. G. A tradução numa perspectiva textual. 1993. Tese (Doutorado em Letras) - Universidade de São Paulo, São Paulo.

XAVIER, R. P. Ensinar e aprender língua estrangeira na contemporaneidade. Contexturas: Ensino crítico de língua inglesa, São José do Rio Preto, v. 17, p.75-94, 2010.

Recebido em: 02/02/2014 Aceito em: 28/04/2014 